\title{
A survey of the prevalence of diabetes type 2 amongst schizophrenics in a chronic care treatment facility
}

AJ Lasich, N Paruk, J Ramparsad

Medical Department, Ekuhlengeni Care Centre, Umbogintwini South Africa

\begin{abstract}
Objective: The determination of the prevalence of type 2 diabetes in a group of patients suffering from schizophrenia who have been hospitalized in a chronic care facility and to consider the role of factors which may contribute to the increased risk of developing diabetes. Method: The hospital files of patients suffering from diabetes type 2 were reviewed and assessments were made in terms of age, gender and body mass index (BMI) duration of stay and co-existing medical conditions. Fasting blood glucose estimations were done on all schizophrenic patients not receiving treatment for diabetes. Medications of patients were recorded. A protocol was prepared and approval to conduct the study was obtained from the hospital authorities. Results: Of 494 schizophrenic patients 19 (3.85\%) were found to suffer from diabetes. $68 \%$ of patients in the diabetic group were found to be overweight in terms of BMI rating. Only one patient had a medical condition. Proportionately the number of women was relatively high. Conclusion: The prevalence if diabetes type 2 in a group of chronically ill hospitalised patients with schizophrenia is significantly lower than in outpatient populations. Residing in a facility long term may serve as a protective factor. The exclusion of psychological stressors such as isolation, poverty and abandonment may account for the finding.
\end{abstract}

Key words: Diabetes type 2; Schizophrenia; Antipsychotic drugs; Risk factors; Hospitalisation

Received: $30-07-2006$

Accepted: 26-09-2006

\section{Introduction}

In recent years there has been a growing interest in the association of the use of atypical antipsychotic agents with the development of type 2 diabetes. Retrospective database analyses have provided evidence that the atypical agents may be associated with the development of diabetes when compared to a general population not on antipsychotic medication. It has also been noted that diabetes in particular may be more common in patients with schizophrenia and bipolar disorder than in the general population. A risk for diabetes is being overweight and the potential for psychotropic agents to increase weight would therefore impact on this health factor. ${ }^{1}$ Following the introduction of phenothiazine's in 1956 the prevalence rates of type 2 diabetes mellitus in patients with schizophrenia showed a marked increase. A prevalence rate of $4.2 \%$ reported by Thonnard - Neumann in 1956 was found to have increased to $17.2 \%$ in 1968. Such cases were referred to in the literature as 'phenothiazine diabetes', 2,3

Risk factors that have been identified as increasing the

\section{Correspondence:}

Prof AJ Lasich

Medical Department, Ekuhlengeni Care Centre

PO Box 3, Umbogintwini 4120 South Africa

email: aj@kwazulunatal.com probability of developing diabetes mellitus include being diagnosed with schizophrenia, being overweight prior to onset of treatment and experiencing a weight increase of more than 10\% during treatment. Demographic factors have included ethnicity (being African American, Hispanic American, Native American, Asian American, Pacific Islander), and a family history of diabetes mellitus. ${ }^{4}$

Norman Sussman (2001) in a review of atypical antipsychotics and weight gain reported that all antipsychotics produce some weight gain but differences existed among the various agents. He referred to a meta-analysis that was performed to estimate the weight change after 10 weeks of treatment at a standard dose of different antipsychotics which revealed that the largest increases occurred with clozapine and olanzapine. ${ }^{5}$

It would seem that from the result of a number of studies, that schizophrenia in itself is a risk factor for diabetes type 2 (Lindenmayer J et al 2001). This risk is enhanced if the patient is prescribed clozapine or olanzapine and to a lesser extent with risperidone, quetiapine and some low-potency conventional antipsychotics. ${ }^{6}$

Studies that report on the effect of antipsychotic medication on weight gain and the emergence of diabetes type 2 rely on health care databases, outpatient populations and to a lesser extent on long term institutionalised patients. As patients especially those with 
schizophrenia require chronic therapy which can be life-long it has been decided to investigate the prevalence of diabetes in a chronic care facility where a substantial number of patients have been hospitalised for many years. The advantage of such a study is the exclusion of certain environmental risk factors for diabetes such as socioeconomic status, increased food availability, substandard living conditions, inadequate nutrition and unhealthy family dynamics.

\section{Method \\ Setting}

Ekuhlengeni Care Centre is a facility of 1100 beds, which admits patients with chronic unremitting psychiatric disorders requiring long term care lasting years if not life long. Those placed at the centre are admitted in terms of the Mental Health Care Act 2002 as involuntary patients and include adult as well as paediatric cases. The paediatric cases presently at the centre were however hospitalized in the past in keeping with previous policy but no longer admitted. Many of the adults with psychotic disorders have had illnesses of ten years or more in duration with multiple admissions to psychiatric hospitals. All patients undergo a medical check-up every 6 months to assess their physical status.

\section{Sample}

The hospital files of all patients were examined in order to establish the correct diagnosis and to exclude disorders other than schizophrenia.

Almost the entire patient population included in the study had been admitted a number of times to psychiatric hospitals and had been suffering from their illness for many years. Thus over time these patients would have been medically assessed repeatedly and possibly subjected to routine investigations on more than one occasion.

\section{Assessment/diagnosis}

Patients diagnosed as suffering from diabetes were assessed in terms of age, gender, body mass index (BMI), duration of stay and co-existing medical conditions.

Diabetes was diagnosed as the result of routine examination, fasting blood glucose estimation and known symptoms such as polydypsia, polyuria, unexplained weight loss, hyperphagia or recurrent infections. The criteria for the diagnosis of diabetes according to the American Diabetes Association are shown in Table I. ${ }^{8}$ Medication prescribed whether neuroleptic or other psychotropic agent was recorded including dosages and route of administration. Fasting blood sugar tests were carried out on schizophrenic patients not receiving treatment for diabetes to ensure that there were no undetected cases.

\section{TABLE I: Criteria for the diagnosis of diabetes mellitus}

1. Symptoms of diabetes plus casual plasma glucose concentration $\geq 200 \mathrm{mg} / \mathrm{dl}(11.1 \mathrm{mmol} / \mathrm{l})$. Casual is defined as any time of day without regard to time since last meal. The classic symptoms of diabetes include polyuria, polydipsia and unexplained weight loss.

2. $F P G \geq 126 \mathrm{mg} / \mathrm{dl}(7.0 \mathrm{mmol} / \mathrm{l})$. Fasting is defined as no caloric intake for at least $8 \mathrm{~h}$

3. 2-h postload glucose $\geq 200 \mathrm{mg} / \mathrm{dl}$ (1 $1.1 \mathrm{mmol} / \mathrm{l})$ during an OGTT. The test should be perfomed as described by WHO using a glucose load containing the equivalent of $75 \mathrm{~g}$ anhydrous glucose dissolved in water.

Abbreviations: $F P G=$ fasting plasma glucose. OGTT $=$ oral glucose tolerance test
Diabetes was monitored by regular monthly fasting blood glucose or by the use of a glucometer when unexpected changes in condition were observed by nursing staff. Those patients with difficult -to-control states were referred to the local provincial hospital.

\section{Results}

At the time of the study there was a total of 1040 patients, of which 494 were diagnosed as having schizophrenia. Specified psychotic disorders other than schizophrenia included 23 with schizoaffective disorder, 7 with bipolar disorder and 1 with delusional disorder. Diagnosis was based on DSM IV criteria. ${ }^{7}$ The gender distribution of those suffering from schizophrenia was 93 women and 401 men. The skewed gender distribution in this group being due to the fact that twice the number of men are accommodated at the centre. As a comparison the schizoaffective disorder group included 14 women and 9 men and the bipolar group 5 women and 2 men (Table II).

\section{TABLE II: Diagnostic categories and gender $(\mathbf{N}=525)$}

\begin{tabular}{|l|l|l|}
\hline Diagnosis & Male & Female \\
\hline Schizophrenic disorder & 401 & 93 \\
Schizoaffective disorder & 9 & 14 \\
Bipolar disorder & 2 & 5 \\
Delusional disorder & 1 & 0 \\
\hline
\end{tabular}

Ages of patients with schizophrenia ranged from 19 to 79 years with a mean of 50 years for women and 47 years for men. Patients suffering from diabetes numbered 28. Their diagnostic categories, gender, age and BMI are listed (Table III).

\section{TABLE III: Diabetic group: demographic data BMI and} diagnosis $(\mathbf{N}=28)$

\begin{tabular}{|l|l|l|l|}
\hline Gender & Age(years) & BMI & Diagnosis \\
\hline Female & 36 & 49.2 & Schizophrenia \\
Female & 28 & 34.6 & M.R with Psychotic disorder N.O.S \\
Female & 42 & 50.8 & Mental Retardation \\
Female & 40 & 26.8 & Schizophrenia \\
Female & 50 & 26.0 & Schizophrenia with M.R \\
Female & 60 & 28.2 & Schizophrenia \\
Female & 34 & 21.2 & Mental Retardation \\
Female & 50 & 31.5 & Schizophrenia \\
Female & 63 & 35.1 & Schizophrenia \\
Female & 48 & 34.1 & Schizophrenia \\
Female & 41 & 25.5 & Schizophrenia with M.R \\
Male & 57 & 27.7 & Schizophrenia with M.R \\
Male & 65 & 32.8 & M.R with Psychotic disorder N.O.S \\
Male & 58 & 25.7 & Dementia \\
Male & 61 & 19.7 & Dementia \\
Male & 55 & 23.3 & Schizophrenia \\
Male & 43 & 23.3 & Schizophrenia \\
Male & 55 & 22.0 & Schizophrenia \\
Male & 56 & 21.7 & Schizophrenia \\
Male & 63 & 29.0 & M.R with Psychotic disorder N.O.S \\
Male & 41 & 24.7 & Schizophrenia \\
Male & 48 & 18.4 & Mental Retardation \\
Male & 62 & 20.0 & Schizophrenia \\
Male & 57 & 28.8 & M.R with Psychotic disorder N.O.S \\
Male & 45 & 29.1 & Schizophrenia \\
Male & 37 & 26.9 & Schizophrenia \\
Male & 47 & 27.2 & Schizophrenia \\
Male & 21 & 36.2 & Schizophrenia \\
\hline & &
\end{tabular}

Abbreviations:

M.R. - Mental Retardation

BMI - Body mass Index

N.O.S - not otherwise specified 
As reflected the group comprised 11 women and 17 men of which 19 suffered from Schizophrenia. This figure represented $3.85 \%$ of the total number of schizophrenic patients at the centre ( $\mathrm{n}=494$ ). BMI ranged from 18.4 to 50.8. In terms of the WHO classification of overweight 1 patient was underweight, 8 were within normal range (28.6\%), 11 pre obese (39.3\%), and 8 severe obese (28.6\%). ${ }^{8}$ Approximately $68 \%$ of patients in the diabetic group were therefore overweight.

Although men outnumbered women in a ratio of $2: 1$ at the centre, $42 \%(n=8)$ were female and 58\% $(n=11)$ male who received treatment for diabetes in the schizophrenic group.

Those suffering from schizophrenia received neuroleptic medication in the form of depot preparations or the oral route twice daily.

The length of stay at the centre of those suffering from diabetes for female patients ranged from 5 to 30 years with a mean of 22 years and for males 2 to 23 years with a mean of 16 years. Hypertension as a co-existing medical condition was found in one female patient with schizophrenia aged 62 years.

Tables IV and V show the list of medications administered to the patients included in the study.

TABLE IV: Medication and age profile of male schizophrenic group with diabetes $(\mathrm{N}=11)$

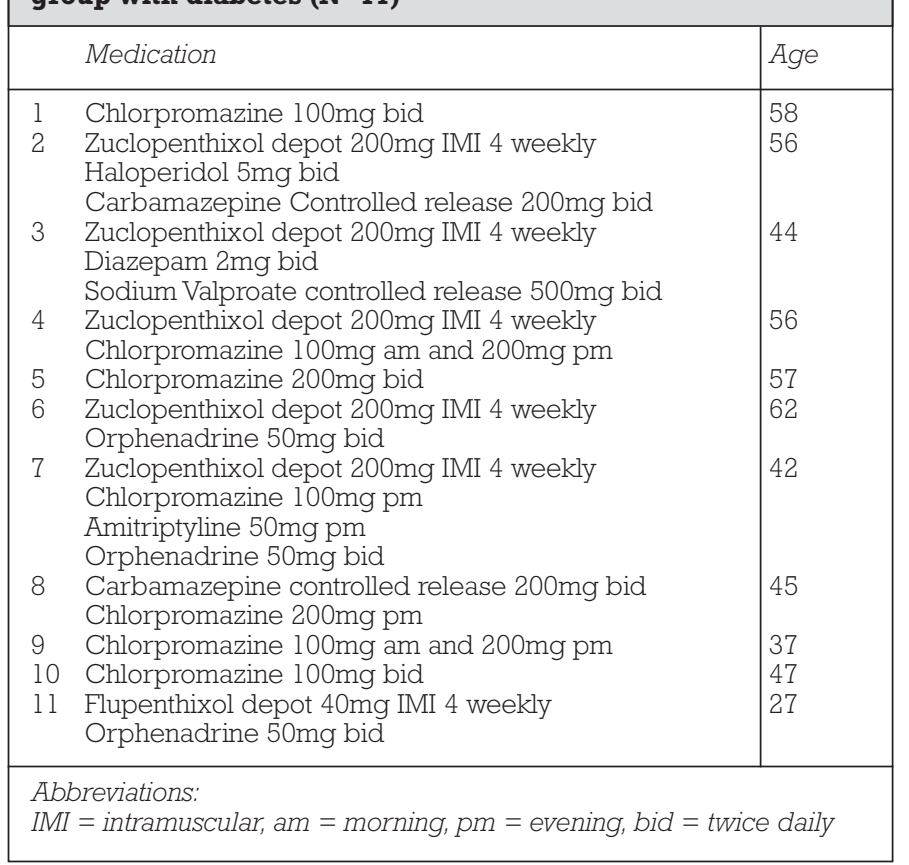

TABLE V: Medication and age profile of female schizophrenic group with diabetes $(\mathbf{N}=8)$

\begin{tabular}{|cl|l|}
\hline & Medication & Age (years) \\
\hline 1 & $\begin{array}{l}\text { Zuclopenthixol depot 200mg IMI 4 weekly } \\
\text { Clozapine 150mg bid }\end{array}$ & 37 \\
2 & Clozapine 100mg bid & 41 \\
3 & $\begin{array}{l}\text { Zuclopenthixol depot 400mg IMI 4 weekly } \\
\text { Clozapine 100mg am and 150mg pm }\end{array}$ & 50 \\
4 & Flupenthixol 60mg IMI 4 weekly & 60 \\
5 & Trifluoperazine 5mg am and 10mg pm & 50 \\
6 & Carbamazepine CR 200mg daily & 63 \\
7 & $\begin{array}{l}\text { Zuclopenthixol depot 200mg IMI 4 weekly } \\
\text { Trifluoperazine 10mg bid }\end{array}$ & 48 \\
8 & Fhlopromazine 50mg bid & 42 \\
\hline
\end{tabular}

Abbreviations:

IMI = intramuscular, am = morning, $p m=$ evening, bid $=$ twice daily

\section{Discussion}

Past studies of diabetes mellitus type 2 in patients with schizophrenia reveal a prevalence rate of between $11 \%$ to $18 \%$. In a more recent and extensive study of United States national schizophrenic samples investigators established a lifetime type 2 diabetes prevalence of $14.9 \%$ and a current prevalence of $10 \%{ }^{9}$ International studies have yielded similar results. In addition there appears to be a significantly higher prevalence rate for female patients and black patients indicating a higher risk for these individuals. The prevalence of diabetes mellitus in the general population has been estimated to be 1.2\% for individuals aged between 18 and 44 years and 6.3\% for those aged 45 to 65 years i.e. according to the 1995 National Health Interview Survey in US. ${ }^{6}$

Our study has shown a surprisingly low prevalence of diabetes type 2 (3.85\%) among the group of patients suffering from chronic schizophrenia. Fasting blood glucose tests on schizophrenics not clinically diabetic did not reveal any latent cases of hyperglycaemia.

Reviews of case reports since 1994 of hyperglycaemia and diabetes mellitus during treatment with atypical antipsychotics have shown that clozapine is the agent most frequently associated with hyperglycaemia followed by olanzapine.In addition it has been found that hyperglycaemia is not dose dependant. ${ }^{10,11}$

The occurrence of hyperglycaemia during treatment with clozapine has been well documented. ${ }^{12,13}$ However in this study only four patients (14,3\%), three female and one male received clozapine. The majority were treated with traditional antipsychotics in combination or as sole agents and at moderate dosages. The pattern of medication prescribed for other patients at the centre follows a similar trend, the majority being on traditional antipsychotics. The extent of the role of antipsychotics as a causative factor is difficult to determine based on our findings or when extrapolated from other studies.

Accepting the fact that known risk factors such as weight gain, gender, being schizophrenic and receiving antipsychotics agents are implicated, the low prevalence in a population of long term hospitalised patients suggests that psychosocial factors may play a role in the development of diabetes type 2 . These factors have been referred to as unhealthy lifestyle habits including inactivity, abuse of alcohol, as well as poverty, poor access to good nutrition, disturbed family dynamics, isolation and abandonment. ${ }^{14}$ The case histories of many admitted to the centre reveal the presence of such factors, which have contributed to relapses and the trend towards chronicity.

Regarding the role of stress in the emergence of diabetes mellitus, Dinan (2004) has developed a model suggesting that patients with schizophrenia show an overactivation of both the hypothalamic - pituitary - adrenal and sympathoadrenal medullary axes. These endocrine systems respond to stress whether acute or chronic by an increase in the production of diabetogenic hormones cortisol and adrenaline. It is proposed that both hormones play a part in the onset of diabetes mellitus in schizophrenia. Dinan makes a distinction between 'true stressors' which emanate from objective space and 'pseudostressors' which are the result of subjective experience of psychotic symptoms. ${ }^{16}$

The limitations of a survey of this nature are that information is based primarily on case reports, retrospective data and clinical assessments. Potential confounding factors such as associated metabolic disorders and environmental influences among others were not explored. 


\section{Conclusion}

This study has revealed a surprisingly low prevalence of diabetes type 2 in a relatively large number of hospitalised patients suffering from chronic schizophrenia and who are severely disabled by their illness. It is possible that investigating the clinically non-diabetic schizophrenic patients by means of an oral glucose tolerance test may have identified other potential cases of hyperglycaemia. Nevertheless known risk factors such as being a sufferer of schizophrenia, weight gain, family history of diabetes and ethnicity have to be weighed against other variables such as socioeconomic status, inactivity and lifestyle habits.

One can postulate that being admitted to a long term facility does provide protective factors that play a significant role in the reduction of diabetes type 2 and associated morbidity in a vulnerable population.

Further studies of a prospective nature using structured interviews and rating scales are needed to examine and allow for the control of such factors as the role of stress in the genesis of diabetes, the effects of environmental influences, associated metabolic disorders and antipsychotic medication.

\section{References}

1. Caro JJ, Ward A, Levinton C. Robinson K. The risk of diabetes during olanzapine use compared with risperidone use: a retrospective database analysis. J Clin Psychiatry 2002; 63: 1135 - 1139.

2. Thonnard - Neumann E. Phenothiazines and diabetes in hospitalised women. Am J Psychiatry 1956; 29: 827 - 828.

3. Thonnard - Neumann E. Phenothiazines and diabetes in hospitalized women. Am J Psychiatry 1968; 124: 978 - 982.

4. Stahl SM. The metabolic syndrome: psychopharmacologists should weigh the evidence for weighing the patient. J Clin Psychiatry 2002; 63: $1094-1095$.

5. Sussman N. Review of atypical antipsychotics and weight gain. J Clin Psychiatry 2001; 62 (suppl 23): 5 - 12.

6. Lindenmayer J, Nathan A, Smith R. Hyperglycaemia associated with the use of atypical antipsychotics. J Clin Psychiatry 2001; 62 (suppl 23): $30-38$.

7. American Psychiatric Association. Diagnostic and Statistical Manual of Mental Disorders, Fourth Edition. Washington, DC: American Psychiatric Association; 1994

8. American Diabetes Association. Diagnosis and classification of diabetes mellitus. Diabetes Care, 2005; 28 (suppl 1): 37 - 42.

9. WHO International obesity task force. Obesity - preventing and managing the global epidemic. Report of a WHO consultation on obesity; Geneva 1997.

10. Dixon L, Weiden P, Delahanty J, et al. Prevalence and correlates of diabetes in national schizophrenic samples. Schizophr Bull 2000; 26 : $903-912$.

11. Sernyak MJ, Leslie DL, Alarcon RD, et al. Association of diabetes mellitus with use of atypical neuroleptics in the treatment of schizophrenia. Am J Psychiatry 2002; 159: 561 - 566

12. Newcomer JW, Haupt DW, Fucetola R, et al. Abnormalities in glucose regulation during antipsychotic treatment of schizophrenia. Arch Gen Psychiatry 2002; 59 : 337 - 345.

13. Henderson DC. Clozapine: diabetes mellitus, weight gain and lipid abnormalities. J Clin Psychiatry 2001; 62 (suppl 23): 39 - 44.

14. Popli AP, Konicki PE, Jurjus GJ, et al. Clozapine and associated diabetes mellitus. J Clin Psychiatry 1997; 58: 108 - 111.

15. Aronne LJ. Epidemiology, morbidity and treatment of overweight and obesity. J Clin Psychiatry 2001; 62 (suppl 23): 13 - 22.

16. Dinan TG. Stress and the genesis of diabetes mellitus in schizophrenia. Br J Psychiatry 2004; 184 (suppl 47) : 72 - 75. 